\title{
UNRAVELING THE SOLVATOCHROMISM OF A TRIARYLMETHANE DYE BY RESONANCE RAMAN SPECTROSCOPY
}

\author{
Natália M. Monezi ${ }^{\mathrm{a}}$, Luiz F. Lepre ${ }^{\mathrm{a}, \mathrm{b}}$ and Rômulo A. Ando ${ }^{\mathrm{a}, *, \bullet}$ \\ anstituto de Química, Departamento de Química Fundamental, Universidade de São Paulo, 05508-000 São Paulo - SP, Brasil \\ 'Laboratoire de Chimie, École Normale Supérieure de Lyon \& CNRS, 69364 Lyon, France
}

Recebido em 26/06/2019; aceito em 04/09/2019; publicado na web em 21/10/2019

\begin{abstract}
The effect of the solvent on the electronic properties of the triarylmethane dye rosolic acid (RA) is investigated by means of UV-VIS and resonance Raman (RR) spectroscopies. The comparison of the solvatochromic behavior of both neutral and dianionic species of RA in acetonitrile, dimethylsulfoxide, and methanol illustrates the effect of polarity and hydrogen bonding on their electronic transitions. The resonance Raman analysis revealed two distinct chromophores in both neutral and dianionic species: $i$ ) one located at the central carbon atom, acting as an electron acceptor, and $i i$ ) the other involving the $\pi$-system of the donor groups. The observed resonance Raman excitation profiles are solvent dependent and could be interpreted as the effect of the solvent on the planarity of the aromatic rings in relation to the central carbon atom. In the case of neutral RA species, having $\mathrm{C}_{2}$ symmetry, specific hydrogen bonding interactions are responsible for inducing a symmetry increase. The opposite effect was observed for the dianionic species $[R A]^{2-}$, having $D_{3}$ propeller symmetry, where the interactions with the solvent induces a lowering of the symmetry.
\end{abstract}

Keywords: rosolic acid; propeller symmetry; solvatochromic probe; hydrogen bonding; crystal violet.

\section{INTRODUCTION}

Triarylmethanes are molecules where a central $\mathrm{sp}^{3}$-hybridized carbon atom is bonded to three aryl groups. Historically, they belong to the group of the first synthetic dyes largely used in textile industry. ${ }^{1}$ Besides their interest for the textile companies, since the beginnings of their commercialization molecules having the triarylmethane motif have found applications in areas such as biological,,${ }^{2,3}$ sensors, ${ }^{4-7}$ dye-cells, ${ }^{8,9}$ and others ${ }^{10-12}$ due to their unique chemical properties.

Beyond their wide commercial interest, the spectroscopic features associated to triarylmethane dyes have attracted much scientific attention due to the large dependence of their electronic spectra with the medium. The solvatochromic properties of triarylmethane dyes were extensively investigated in the past due to their UV-VIS absorption maxima dependence with the solvent, ${ }^{13,14}$ concentration ${ }^{15}$ and temperature. ${ }^{16}$ Amongst the triarylmethane dyes the most explored is Crystal Violet (CV), where three identical dimethylamine electron donor groups are connected to a central electron acceptor carbon atom with a partially positive charge. Due to the steric hindrance associated to aromatic rings, the molecular configuration of $\mathrm{CV}$ and other symmetrical triarylmethane dyes is characterized by blade propeller symmetry ( $\mathrm{D}_{3}$ point group). ${ }^{1}$

In the UV-VIS spectra of CV the appearance of a shoulder on the higher energy side of the main absorption band was subject of intense debate in the scientific literature. Lewis et al. ${ }^{17}$ proposed the existence of two ground state rotational isomers in equilibrium having $D_{3}$ and $C_{2}$ symmetries due to the orientation of the aromatic rings. Several experimental and theoretical works have challenged such model and different propositions are available in the literature, where there is still no consensus about this subject. The origin of such shoulder has been attributed to solvent interactions, ${ }^{13,14,16,18}$ aggregation, ${ }^{15}$ ion-pair formation, ${ }^{19}$ or the existence of two ground states in solution. ${ }^{20,21}$ The historical controversies involving the CV shoulder can be found in a pedagogical paper by Kahr et al. ${ }^{22}$ where the authors, using X-Ray

*e-mail: raando@iq.usp.br crystal structure determination, showed that the two prevailing theories are in fact not mutually exclusive, i.e. $i$ ) the existence of two isomers in equilibrium and $i$ ) the existence of two excited states by the lowering of symmetry induced by interactions.

The first papers using resonance Raman spectroscopy to tackle this issue concluded that the shoulder was a result of a vibronic structure of a single electronic state. ${ }^{23,24}$ After that, McHale et al. using resonance Raman depolarization ratios, fluorescence spectra and theoretical calculations, proposed that the interactions of $\mathrm{CV}$ with solvent or ions were responsible for lowering the $\mathrm{D}_{3}$ symmetry resulting in the splitting of the first excited state. ${ }^{14,25}$ As pointed by Kahr et al. ${ }^{22}$ since only the Raman depolarization ratios, and not the normal modes assignment, differed from that expected for a $D_{3}$ symmetry, the conclusion was that the symmetry breaking induced by interactions was electronic rather than mechanical. In the same paper Kahr et al. showed that Raman spectroscopy was unable to distinguish between to $\mathrm{CV}$ hydrates in different crystalline symmetry groups. ${ }^{22}$ More recently Loison et al. ${ }^{26}$ showed that the shoulder is due to interactions of $\mathrm{CV}$ with its environment, by presenting the photodissociation spectra for isolated $\mathrm{CV}$ and $\mathrm{CV}-\mathrm{H}_{2} \mathrm{O}$ complex without and with a shoulder, respectively.

The present work studies the solvatochromic behavior of the triarylmethane dye Rosolic Acid (RA, Figure 1) in solvents having distinct dielectric constants. The rationale for choosing RA is due to its $\mathrm{C}_{2}$ symmetry as a neutral species, while in a basic medium it assumes the $\mathrm{D}_{3}$ propeller symmetry due to deprotonation of its $-\mathrm{OH}$ groups (Figure 1). The solvatochromic behavior of RA was characterized by UV-VIS and the chromophoric groups were characterized by resonance Raman (RR) spectroscopy. To the best of our knowledge, although many different triarylmethane dyes were spectroscopically characterized in the past, this is the first Raman characterization of Rosolic Acid.

\section{EXPERIMENTAL}

Both rosolic acid (RA) and the solvents were purchased from Sigma-Aldrich (>98\%) and were used without further purification. 


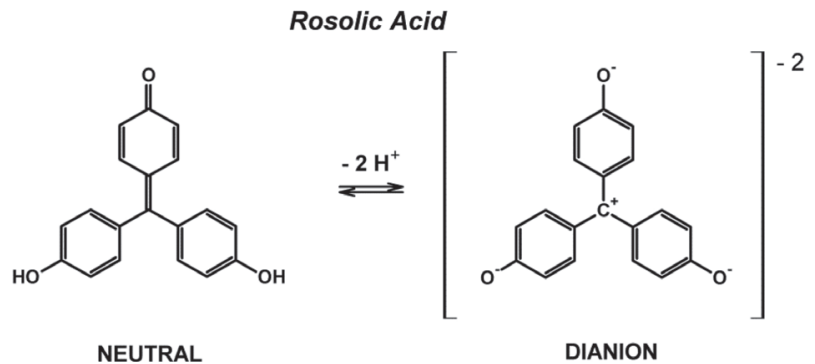

Figure 1. Acid base equilibrium between neutral and dianionic structures of rosolic acid

In order to have the same final concentration of RA in each solution $\left(1.0 \times 10^{-5} \mathrm{~mol} \mathrm{~L}^{-1}\right)$, stock solutions of $3.0 \times 10^{-3} \mathrm{~mol} \mathrm{~L}^{-1}$ of the dianionic form of RA were prepared using basic solutions consisting of mixtures of $99 \%$ of the solvent with $1 \%$ of saturated $\mathrm{KOH}$ in water. The protonation/deprotonation of the rosolic acid could be controlled by UV-VIS spectroscopy as a function of the amount of $\mathrm{KOH}$ saturated water added to the solvent. The proportion of solvent $(99 \%) / \mathrm{KOH}_{(\mathrm{aq})}(1 \%)$ was chosen to ensure that only one species was observed in the UV-VIS spectra of the RA solution. The solution of the neutral RA in methanol was obtained adding 5 drops of aqueous hydrochloric acid in $10 \mathrm{~mL}$ of its basic solution. The solutions of neutral RA in acetonitrile and DMSO were prepared separately.

The UV-VIS spectra were collected in a Shimadzu UV3101PC spectrophotometer using a $1 \mathrm{~mm}$ optical path quartz cuvette. Resonance Raman spectra of the neutral and the anionic RA solutions were measured in a Horiba Jobin-Yvon T64000 triple monochromator spectrometer with a CCD detector cooled by liquid nitrogen using radiations at 647.1, 568.2, 530.9, 514.5, 488 and $457.9 \mathrm{~nm}$ (Laser Coherent Innova $70 \mathrm{C} \mathrm{Ar}^{+} / \mathrm{Kr}^{+}$). The samples were placed in adapted NMR tubes and the spectra were acquired using a rotator shaft in order to minimize local heating or eventual photodegradation.

The ground state geometries of RA in its neutral and anionic forms were obtained from Density Functional Theory (DFT) using B3LYP functional ${ }^{27}$ and the $6-311++\mathrm{G}(\mathrm{d}, \mathrm{p})$ atomic basis set. The geometry optimization and vibrational frequency calculations were performed using the Gaussian 09 software..$^{28}$ The SMD (solvation model based on density $)^{29}$ model was considered for all the procedures. No imaginary frequencies were found in the vibrational frequency analyses, indicating that the optimized geometries were in a minimum of the potential energy surface.

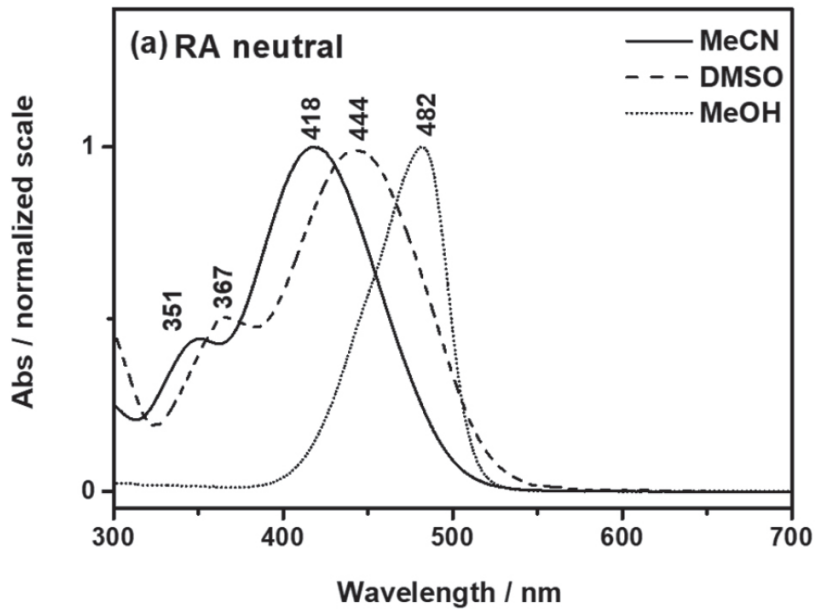

\section{RESULTS AND DISCUSSION}

The electronic absorption spectra of neutral RA in acetonitrile $(\mathrm{MeCN})$, dimethylsulfoxide (DMSO) and methanol $(\mathrm{MeOH})$ solutions are presented in Figure 2a. It can be observed that the band maximum absorption and also the band lineshape are solvent dependent. Since the resonance Raman (RR) effect is related to high values of molar absorption coefficients, the present work will focus on the most intense RA bands observed at $\lambda>400 \mathrm{~nm}$, bands that can be assigned to a $\pi-\pi *$ electronic transition.

In the aprotic solvents the band shapes are similar to each other, presenting a redshift from $418 \mathrm{~nm}$ in $\mathrm{MeCN}$ to $444 \mathrm{~nm}$ in DMSO. The absorption spectra will be explained herein considering the solvent dielectric constant $(\varepsilon)$ since solute-solvent interaction plays a major role in defining the energetic profile in solvents with $\varepsilon>\sim 10 .{ }^{19}$ Therefore, considering the dielectric constant of $\mathrm{MeCN}(\varepsilon=36.64)$ and of DMSO ( $\varepsilon=46.70)$, these UV-VIS results show that an increase in medium polarity leads to a lower energy of electronic transition. These results suggest also that DMSO stabilizes the excited electronic state better than $\mathrm{MeCN}$, indicating that the excited state of neutral $\mathrm{RA}$ is more polar than its ground electronic state.

In the case of the protic solvent $\mathrm{MeOH}$, Figure 2a shows that the spectrum of neutral RA shows a distinct behavior. The UV-VIS band is significantly redshifted $(482 \mathrm{~nm})$ in comparison to the aprotic solvents, even with $\mathrm{MeOH}$ having a dielectric constant that is lower $(\varepsilon=32.63)$ than those of MeCN and DMSO. Besides the significant band shift, it is clearly observed a distinct band lineshape in $\mathrm{MeOH}$ in comparison to that observed in the other two aprotic solvents. In $\mathrm{MeOH}$ the band is narrower and presents a shoulder on the higher energy side, a lineshape that is comparable to that observed for the most studied triarylmethane crystal violet. ${ }^{18} \mathrm{~A}$ possible explanation for this lineshape is to consider that RA has a quinone group susceptible to hydrogen bond the $\mathrm{OH}$ group of methanol molecule. The interaction with $\mathrm{MeOH}$ leads to a larger stabilization of the neutral RA, mainly of the excited state, decreasing the transition energy. These results will be discussed again later together along the resonance Raman data.

When RA is doubly deprotonated, $[\mathrm{RA}]^{2-}$, the negative charges are delocalized within the $\pi$-system and the three rings become identical, characterizing the propeller $\mathrm{D}_{3}$ symmetry as other symmetrical triarylmethane dyes (Figure 1). The UV-VIS spectra of the [RA $]^{2-}$ in $\mathrm{MeCN}, \mathrm{DMSO}$ and $\mathrm{MeOH}$ are shown in Figure 2b. As well as for the neutral RA, the present work will be focused on discussing the most intense bands of the deprotonated dye observed at $\lambda>400 \mathrm{~nm}$.

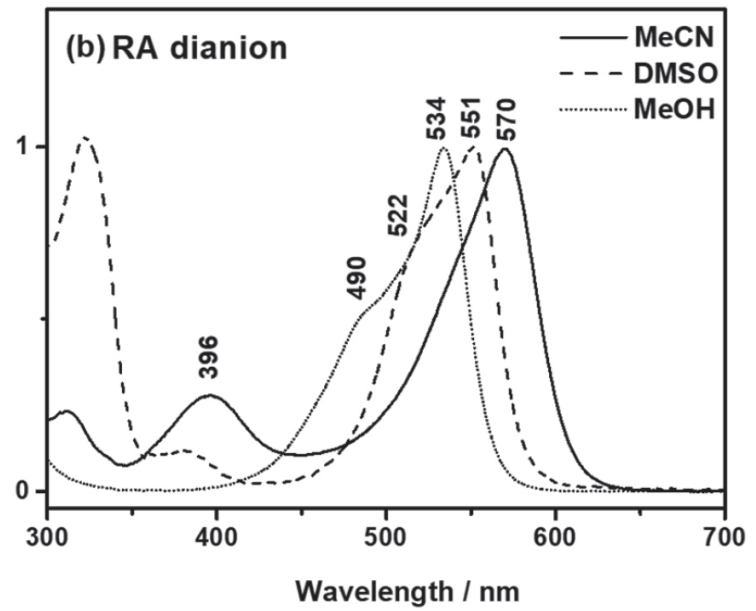

Figure 2. UV-VIS spectra of neutral (a) and dianionic (b) species of rosolic acid (RA) in acetonitrile (MeCN), dimethylsulfoxide (DMSO) and methanol (MeOH) solutions 
In comparison to the neutral RA, as one could expect, the deprotonation of both $-\mathrm{OH}$ groups leads to a substantial band shift to lower energies, as well as an increased molar absorptivity coefficient, regardless the solvent. The negative charges in the oxygen atoms of $[\mathrm{RA}]^{2-}$ (Figure 1) causes a higher electronic delocalization leading to electronic transitions of lower energy. However, in contrast to the behavior observed for neutral RA, the largest redshift was observed for the solvent having the lowest dielectric constant value $(\mathrm{MeCN})$. This behavior is not surprising if [RA] $]^{2-}$ is compared with other charged dyes. Since the ground electronic state of the deprotonated [RA] $]^{2-}$ is doubly charged, then probably there is a higher destabilization of the ground state in less polar solvents leading to a decrease of the HOMO-LUMO gap, as occurs in other ground state charged dyes. ${ }^{30}$ It would be the reason to the observation of a solvatochromic reverse order in comparison to the neutral species.

Relative to the band lineshape, the absorption spectrum of [RA $]^{2-}$ in $\mathrm{MeOH}$ reveals once again a very distinct behavior in comparison to that in the aprotic solvents MeCN and DMSO. The electronic transition energy presented the highest energy value $(\lambda \approx 534 \mathrm{~nm})$ between the three solvents, and a very pronounced shoulder at the higher energy side of the band $(\lambda \approx 490 \mathrm{~nm})$. In this case, in comparison to neutral RA, one can suppose that the specific hydrogen bond between $[\mathrm{RA}]^{2-}$ and $\mathrm{MeOH}$ may induce a lowering of $\mathrm{D}_{3}$ symmetry. These UV-VIS results for [RA] ${ }^{2-}$ support the previous hypothesis of solvent specific interaction used to justify the presence of the higher-energy shoulder observed in $\mathrm{CV}$ absorption spectrum. ${ }^{14,26}$ Aiming to better support such assumptions, a resonance Raman investigation was done.

Prior to the resonance Raman analysis it is important to discuss a few aspects of the Raman spectra of both RA and [RA] ${ }^{2-}$ species. DFT calculations were performed for both species and the theoretical Raman spectra were obtained. A good agreement between the experimental and the theoretical Raman spectra is observed for both neutral RA and dianionic [RA $]^{2-}$ considering acetonitrile as a solvent. Larger discrepancies were observed when the other two solvents DMSO and $\mathrm{MeOH}$ were considered. Therefore, aiming to confirm the vibrational assignments of the neutral RA and the dianionic $[\mathrm{RA}]^{2-}$ Raman spectra, only the DFT calculations for $\mathrm{MeCN}$ is presented. Figure 3 compares the experimental Raman spectra of both RA and $[\mathrm{RA}]^{2-}$ in acetonitrile $(\mathrm{MeCN})$ with their respective theoretical Raman spectra obtained via DFT calculations. Table 1 presents the wavenumbers of the most characteristic Raman bands and their respective assignment, considering the present DFT calculations and the literature for the triarylmethane crystal violet. ${ }^{23,25,31}$

For the neutral RA, Figure 3 shows that the most intense bands appear at $1625,1595,1514$ and $1483 \mathrm{~cm}^{-1}$ and can be assigned to $\phi(8 \mathrm{a})$ $+v(\mathrm{C}=\mathrm{O}), \phi(8 \mathrm{a}), v(\mathrm{C}=\phi)+\phi(19 \mathrm{a})$ and $v(\mathrm{C}=\phi)+\phi(19 \mathrm{~b})$, respectively. The $v\left(C-\phi_{3}\right)$ breathing mode appears at $254 \mathrm{~cm}^{-1}$ and is in agreement with phenolic rings as substituents, as this mode is sensitive to the mass of the substituent groups. ${ }^{23}$ The neutral RA species have $\mathrm{C}_{2}$ symmetry so that the vibrational assignment is relatively simple and the analysis considering the literature for quinone and phenolic rings is well suited for the present case.

The deprotonation of both $-\mathrm{OH}$ groups leads the symmetry to change from $\mathrm{C}_{2}$ to $\mathrm{D}_{3}$ point group and the intensity pattern of the resulting $[\mathrm{RA}]^{2-}$ is significantly different from that of the neutral RA (Figure 3). The characteristic vibrational mode $(\mathrm{C}=\mathrm{O})$ observed at $1625 \mathrm{~cm}^{-1}$ in the neutral RA spectrum appears at $1522 \mathrm{~cm}^{-1}$ in the dianionic $[\mathrm{RA}]^{2-}$ spectrum. The other characteristic vibrational mode $(\mathrm{C}=\phi)$ observed at $1483 \mathrm{~cm}^{-1}$ in the RA spectrum is rather seen at $1354 \mathrm{~cm}^{-1}$ in the $[\mathrm{RA}]^{2-}$ spectrum. The $v\left(\mathrm{C}-\phi_{3}\right)$ breathing mode is shifted to higher wavenumbers, from $254 \mathrm{~cm}^{-1}$ in neutral RA to 265 $\mathrm{cm}^{-1}$ in the anionic $[\mathrm{RA}]^{2-}$. All these wavenumber shifts agree with the higher electronic delocalization of the dianionic species $[\mathrm{RA}]^{2-}$, as observed in the UV-VIS spectra (Figure 2). The other vibrational modes, most of them related to ring modes, change slightly with the dideprotonation in terms of wavenumber shifts, whereas their intensities are drastically changed. These intensity changes will be discussed further in the analysis of the resonance Raman data, as follows.

The Raman spectra of neutral RA in MeCN, DMSO and MeOH solutions were measured using different excitation laser lines are
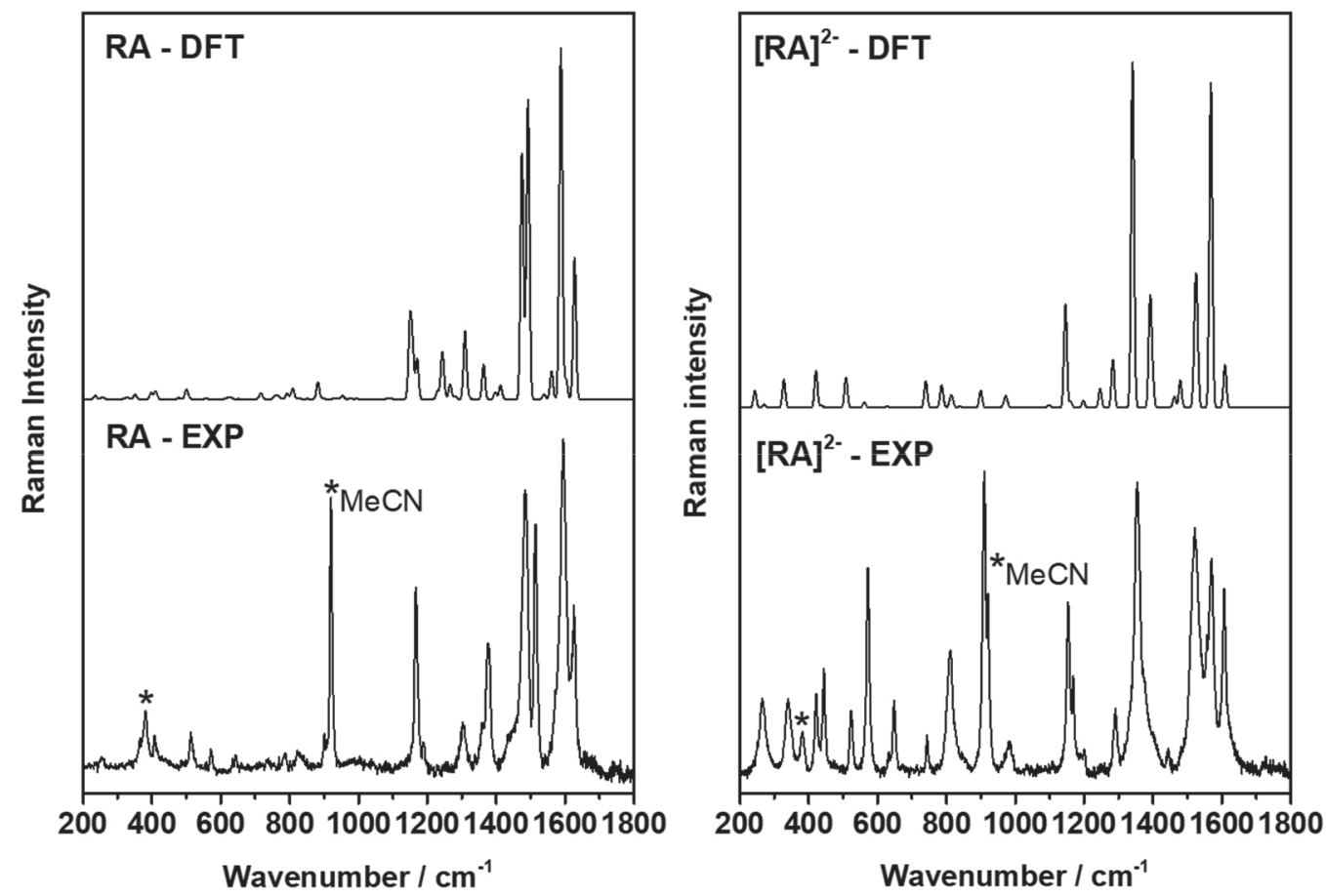

Figure 3. Experimental (EXP, bottom) and theoretical (DFT, top) Raman spectra of neutral RA (left) and dianionic [RA ${ }^{2-}$ (right) in acetonitrile (MeCN). *Solvent bands are marked with an asterisk 
Table 1. Experimental (EXP) and theoretical (DFT) Raman wavenumbers and vibrational assignments of neutral and dianionic species of rosolic acid (RA) in acetonitrile (MeCN), dimethylsulfoxide (DMSO) and methanol $(\mathrm{MeOH})$

\begin{tabular}{|c|c|c|c|c|c|}
\hline \multirow{2}{*}{ Species } & \multicolumn{2}{|c|}{$\mathrm{MeCN}$} & \multirow{2}{*}{$\frac{\text { DMSO }}{\text { EXP }}$} & \multirow{2}{*}{$\frac{\mathrm{MeOH}}{\mathrm{EXP}}$} & \multirow{2}{*}{$\begin{array}{l}\text { Vibrational } \\
\text { assigment }^{\mathrm{a}}\end{array}$} \\
\hline & EXP & DFT & & & \\
\hline \multirow{16}{*}{ RA } & 254 & 235 & 257 & 256 & $\mathrm{C}-\phi_{3}$ breathing \\
\hline & & 398 & 384 & 361 & OP $\phi(16 a)$ \\
\hline & 408 & 411 & 410 & 411 & OP $\phi(16 b)$ \\
\hline & 513 & 500 & 517 & 516 & $\mathrm{OP} \phi(11)$ \\
\hline & 644 & 625 & & 646 & $\phi(6 a)$ \\
\hline & 787 & 793 & 788 & 795 & OP $\phi(4)$ \\
\hline & 824 & 809 & & & $\phi(1)+\phi(10 a)$ \\
\hline & 901 & 882 & 902 & 905 & $\phi(12)$ \\
\hline & 1167 & 1151 & 1164 & 1166 & $\phi(9 a)$ \\
\hline & 1189 & 1170 & 1189 & 1193 & $\phi(9 b)$ \\
\hline & 1302 & 1309 & 1311 & 1299 & $\phi(14)$ \\
\hline & 1376 & 1363 & 1380 & 1378 & $\phi(15)$ \\
\hline & 1483 & 1475 & 1480 & 1464 & $\mathrm{C}=\phi+\phi(19 \mathrm{a})$ \\
\hline & 1514 & 1492 & 1512 & 1512 & $\mathrm{C}=\phi+\phi(19 b)$ \\
\hline & 1595 & 1588 & 1595 & 1594 & $\phi(8 a)$ \\
\hline & 1625 & 1628 & 1623 & 1617 & $\phi(8 a)+v(C=O)$ \\
\hline \multirow{20}{*}[\mathrm{RA}]{$^{2-}$} & 265 & 244 & 260 & 270 & $\mathrm{C}-\phi_{3}$ breathing \\
\hline & 340 & 328 & 335 & 345 & $\mathrm{OP} \phi(16 \mathrm{a})$ \\
\hline & 422 & 421 & 423 & 421 & OP $\phi(16 b)$ \\
\hline & 444 & 456 & 445 & 443 & $\delta\left(\mathrm{C}=\mathrm{O}^{-}\right)$ \\
\hline & 523 & 508 & 520 & 525 & OP $\phi(11)$ \\
\hline & 572 & 562 & 568 & 574 & $\phi(6 a)+\phi(17 b)$ \\
\hline & 648 & 627 & 645 & 652 & $\phi(6 a)$ \\
\hline & 743 & 740 & 743 & 744 & OP $\phi(4)$ \\
\hline & 810 & 815 & 809 & 812 & $\phi(1)$ \\
\hline & 901 & 900 & 907 & 913 & $\phi(12)+\delta\left(\mathrm{C}=\phi_{3}\right)$ \\
\hline & 984 & 972 & 981 & 993 & $\phi(12)$ \\
\hline & 1153 & 1146 & 1152 & 1156 & $\phi(9 a)$ \\
\hline & 1168 & 1161 & 1165 & 1172 & $\phi(9 b)$ \\
\hline & 1291 & 1283 & 1291 & 1298 & $\phi(3)$ \\
\hline & 1354 & 1341 & 1351 & 1358 & $\mathrm{C}=\phi+\phi(19 \mathrm{a})$ \\
\hline & 1375 & 1392 & 1397 & 1373 & $\phi(19 a)$ \\
\hline & 1446 & 1462 & 1440 & 1449 & $\phi(15)$ \\
\hline & 1522 & 1525 & 1529 & 1522 & $v\left(\mathrm{C}=\mathrm{O}^{-}\right)+\phi(19 \mathrm{a})$ \\
\hline & 1570 & 1568 & 1569 & 1570 & $\phi(8 a)+v\left(C=\mathrm{O}^{-}\right)$ \\
\hline & 1607 & 1609 & 1605 & 1609 & $\phi(8 a)$ \\
\hline
\end{tabular}

aRing modes are assigned in Varsanyi notation.

shown in Figure 4. The spectra in $\mathrm{MeCN}$ excited at $\lambda_{0}=488 \mathrm{~nm}$ (Figure 4a) shows that at preresonance condition several bands are enhanced, especially the band assigned to the $v\left(\mathrm{C}-\phi_{3}\right)$ breathing mode at $254 \mathrm{~cm}^{-1}$. On the other hand, such breathing mode is not at all enhanced at resonance condition $\left(\lambda_{0}=457 \mathrm{~nm}\right)$, while other bands at higher frequencies are preferentially enhanced, especially those associated to the quinone ring. This behavior is observed to be essentially the same in DMSO solutions (Figure $4 \mathrm{~b}$ ) since the spectrum excited at $\lambda_{0}=514 \mathrm{~nm}$ shows a selective enhancement of the band at $257 \mathrm{~cm}^{-1}$, while the enhancement of quinone bands at higher frequencies is more pronounced in the spectrum excited at $\lambda_{0}=488 \mathrm{~nm}$. In fact, one could expect very similar resonance Raman enhancement profiles for neutral RA in MeCN and DMSO due to the similarity of the electronic bands observed in the UV-VIS analysis (Figure 2), the difference being the excitation energy in which the Raman spectrum is most enhanced due to the solvatochromic effect.

For the neutral RA in methanol solutions Figure 4 shows that at preresonance condition $\left(\lambda_{0}=514 \mathrm{~nm}\right)$ the $v\left(C-\phi_{3}\right)$ breathing mode is also selectively enhanced at $256 \mathrm{~cm}^{-1}$. However, in contrast to what was observed in $\mathrm{MeCN}$ and DMSO solutions, a more pronounced enhancement of the bands at $361,411,516$ and $795 \mathrm{~cm}^{-1}$ was observed. These bands were assigned to out of plane ring modes $\phi(16 a), \phi(16 b)$, $\phi(11)$ and $\phi(4)$, respectively. It is worth mentioning that these bands assigned to out of plane ring modes are already enhanced in the preresonance Raman spectra in $\mathrm{MeCN}$ and DMSO, but in a lesser extent (Figure 4). Therefore, it is clear that in $\mathrm{MeOH}$ solutions there is a significant perturbation of the symmetry of RA in comparison to RA in the other polar aprotic solvents. At resonance condition $\left(\lambda_{0}=457 \mathrm{~nm}\right)$ the quinone ring bands are enhanced, as well as observed for MeCN and DMSO solvents.

Thus, the resonance Raman analysis of neutral RA in MeCN, DMSO and $\mathrm{MeOH}$ solutions supports the idea of the presence of two distinct excited states of similar energies. We can conclude that the Franck-Condon factors associated with each transition are modulated by the interaction with the medium, determining the resonance Raman enhancement profiles. Such feature will reveal to be even more evident in the analysis of the resonance Raman excitation profile of $[\mathrm{RA}]^{2-}$ species.

The resonance Raman spectra of the dianion $[\mathrm{RA}]^{2-}$ in $\mathrm{MeCN}$, DMSO and $\mathrm{MeOH}$ solutions are shown in Figure 5. In the case of MeCN (Figure 5a), upon using as excitation radiation $\lambda_{0}=568 \mathrm{~nm}$ our system is at strict resonance condition $\left(\lambda_{\max }=570 \mathrm{~nm}\right.$, Figure $\left.2 \mathrm{~b}\right)$ and we are measuring the Raman spectrum of the lowest electronic transition energy and the highest intensity UV-VIS band. It is observed a huge enhancement of the bands at 265 and $444 \mathrm{~cm}^{-1}$, assigned to the $\left(\mathrm{C}-\phi_{3}\right)$ breathing mode and the $\left(\mathrm{CO}^{-}\right)$, respectively. On the other hand, when the same solution is excited at $\lambda_{0}=514 \mathrm{~nm}$ (Figure 5a), i.e. in resonance with the shoulder at ca. $\lambda_{\max }=530 \mathrm{~nm}$ (Figure 2b) the phenolate quinoid modes, e.g. $v(\mathrm{C}=\phi)+\phi(19 \mathrm{a})$ at $1354 \mathrm{~cm}^{-1}$ and $\phi(8 \mathrm{a})$ at $1607 \mathrm{~cm}^{-1}$, are selectively enhanced.

The Raman spectra in different resonance conditions of $[R A]^{2-}$ in DMSO (Figure 5b) showed a similar behavior as [RA] $]^{2-}$ in $\mathrm{MeCN}$, i.e. the bands at 260 and $445 \mathrm{~cm}^{-1}$ were significantly enhanced when the spectrum is excited at $\lambda_{0}=568 \mathrm{~nm}$, while the bands at 1351 and $1605 \mathrm{~cm}^{-1}$ were enhanced upon excitation at $\lambda_{0}=514 \mathrm{~nm}$.

As expected from the UV-VIS spectra, the resonance Raman excitation profile of $[\mathrm{RA}]^{2-}$ in $\mathrm{MeOH}$ revealed a distinct pattern in comparison to other two solvents. It can be noticed in the spectrum obtained at $\lambda_{0}=568 \mathrm{~nm}$ (Figure $5 \mathrm{c}$ ) that besides the enhancement of both bands at 270 and $443 \mathrm{~cm}^{-1}$, the enhancement of several bands assigned to ring modes at $574,652,812,913 \mathrm{~cm}^{-1}$ is also observed. When the spectrum of $[\mathrm{RA}]^{2-}$ in $\mathrm{MeOH}$ is excited at resonance $\left(\lambda_{0}=\right.$ $488 \mathrm{~nm}$ ) with the shoulder at $\lambda_{\max }=490 \mathrm{~nm}$ (Figure $2 \mathrm{~b}$ ), the phenolate quinoid ring modes are enhanced, similarly to what was observed for $[\mathrm{RA}]^{2-}$ in MeCN and DMSO.

The concomitant analysis of UV-VIS and resonance Raman data for the $[\mathrm{RA}]^{2-}$ in the three solvents shows that the closer in energy are the transitions (Figure 2), the better the distinction between the two chromophores (Figure 5). The present investigation for rosolic acid agrees with the interpretation of McHale et al. ${ }^{14}$ that the high-energy shoulder observed in crystal violet spectra is more evident when 

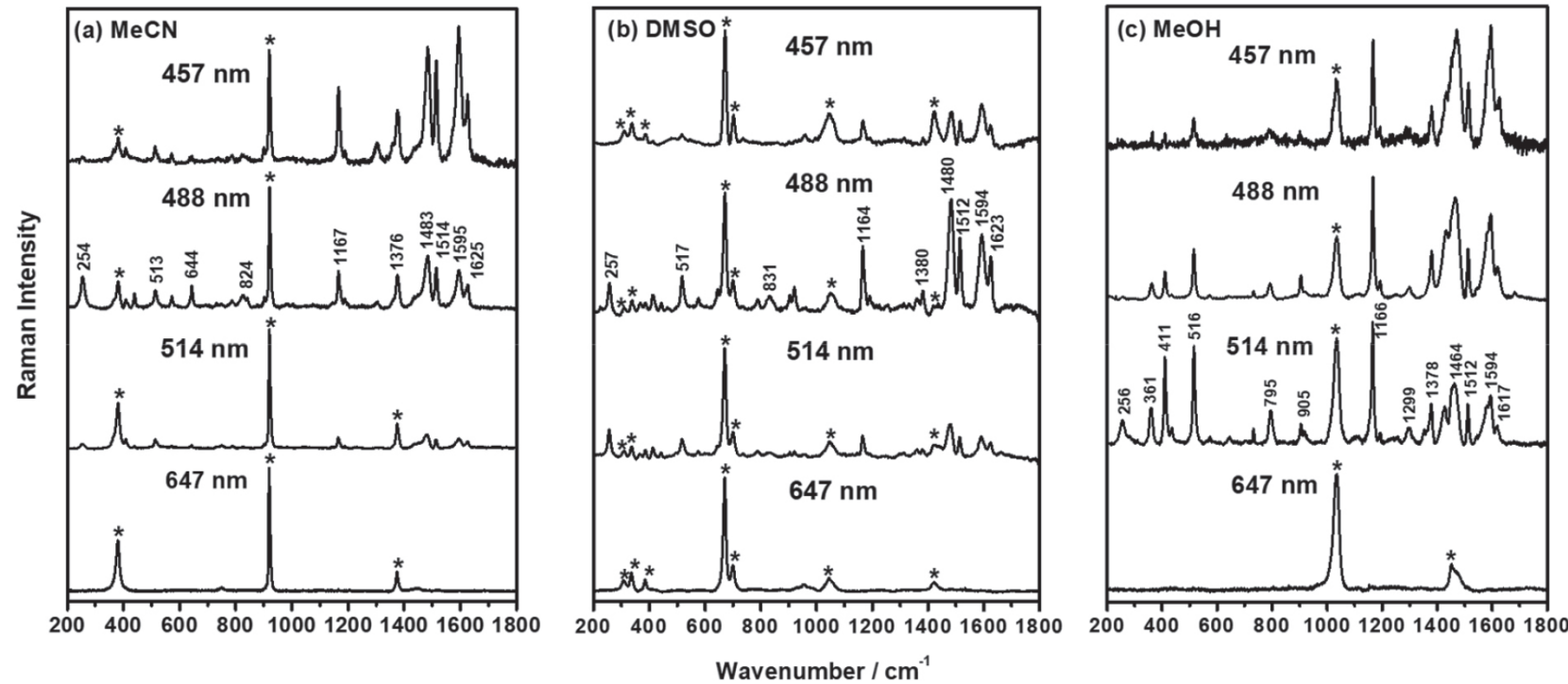

Figure 4. Resonance Raman spectra of neutral RA in (a) acetonitrile, MeCN, (b) dimethylsulfoxide, DMSO, and (c) methanol, MeOH solutions. *Solvent bands are marked with an asterisk
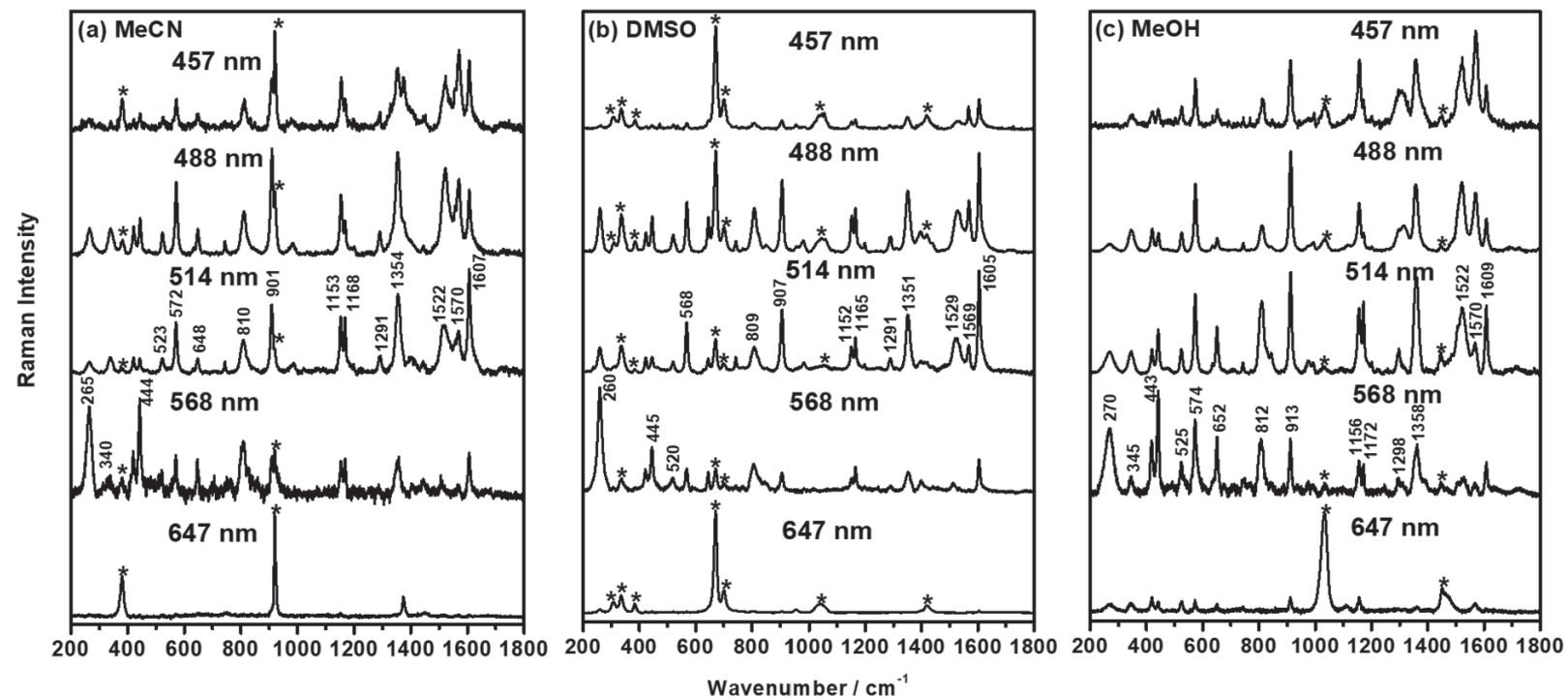

Figure 5. Resonance Raman spectra of dianion $[R A]^{2-}$ in (a) acetonitrile, MeCN, (b) dimethylsulfoxide, DMSO, and (c) methanol, MeOH solutions. *Solvent bands are marked with an asterisk

there is a greater perturbation by solvent molecules, responsible for the lowering from $\mathrm{D}_{3}$ symmetry to $\mathrm{C}_{2}$. Therefore, we can interpret the $[\mathrm{RA}]^{2-}$ in $\mathrm{MeCN}$ has a higher symmetry than in $\mathrm{MeOH}$ due to the specific interaction of the last with $[\mathrm{RA}]^{2-}$.

\section{CONCLUSIONS}

The solvatochromic properties of the triarylmethane dye rosolic acid (RA) were investigated by UV-VIS and resonance Raman spectroscopy. The perturbation of the symmetry of the neutral RA and of the dianion [RA $]^{2-}$ could be addressed, particularly in methanol solutions due to specific hydrogen bonding interactions. In the case of neutral RA the symmetry increases from $\mathrm{C}_{2}$ to $\mathrm{D}_{3}$ with methanolic solvation, while in the case of $[\mathrm{RA}]^{2-}$ the opposite effect was observed, i.e. the symmetry is lowered to $C_{2}$ due to specific interactions with methanol. The results presented herein support the single ground state model with the split of the excited $\mathrm{S}_{1}$ state due to the lift of degeneracy induced by the interaction with solvent molecules, similarly to the classical case of crystal violet $(\mathrm{CV})$. Different from the $\mathrm{CV}$ resonance Raman enhancement profiles, in the case of RA it was possible a clear distinction of the two chromophores in solution.

\section{ACKNOWLEDGEMENTS}

N.M.M. thanks the Coordenação de Aperfeiçoamento de Pessoal de Nível Superior for the fellowship (CAPES 132249/2012-0). L.F.L. thanks Fundação de Amparo à Pesquisa do Estado de São Paulo (FAPESP 2013/23234-7) for the fellowship. R. A. A. thanks CNPq (306690/2015-2) for the fellowship and FAPESP grant $(2016 / 21070-5)$

\section{REFERENCES}

1. Duxbury, D. F.; Chem. Rev. 1993, 93, 381.

2. Eldem, Y.; Ozer, I.; Dyes Pigm. 2004, 60, 49.

3. Jang, M. S.; Kang, N. Y.; Kim, K. S.; Kim, C. H.; Lee, J. H.; Lee, Y. C.; FEMS Microbiol. Lett. 2007, 271, 78. 
4. Gotor, R.; Costero, A. M.; Gil, S.; Parra, M.; Martinez-Manez, R.; Sancenon, F.; Chem. - Eur. J. 2011, 17, 11994.

5. Gotor, R.; Costero, A. M.; Gil, S.; Parra, M.; Martinez-Manez, R.; Sancenon, F.; Gavina, P.; Chem. Commun. 2013, 49, 5669.

6. Matsui, M.; Kakitani, K.; Kubota, Y.; Funabiki, K.; Ishida, M.; RSC Adv. 2016, 6, 16759.

7. Zavakhina, M. S.; Yushina, I. V.; Samsonenko, D. G.; Dybtsev, D. N.; Fedin, V. P.; Argent, S. P.; Blake, A. J.; Schroder, M.; Dalton Trans. 2017, 46, 465 .

8. Chitambar, M.; Wang, Z. J.; Liu, Y. M.; Rockett, A.; J. Am. Chem. Soc. 2012, 134, 10670 .

9. Ng, C. H.; Ohlin, C. A.; Winther-Jensen, B.; Dyes Pigm. 2015, 115, 96.

10. Bartholome, D.; Klemm, E.; Macromolecules 2006, 39, 5646.

11. Ley, C.; Brendle, J.; Walter, A.; Jacques, P.; Ibrahim, A.; Allonas, X.; PCCP 2015, 17, 16677.

12. Kian, R.; Zakerhamidi, M. S.; Shamkhali, A. N.; Nesari, P.; J. Mol. Liq. 2017, 225, 653 .

13. Korppitommola, J.; Yip, R. W.; Can. J. Chem. 1981, 59, 191.

14. Lueck, H. B.; McHale, J. L.; Edwards, W. D.; J. Am. Chem. Soc. 1992, $114,2342$.

15. Lueck, H. B.; Rice, B. L.; McHale, J. L.; Spectrochim. Acta, Part A 1992, 48, 819 .

16. Korppitommola, J.; Kolehmainen, E.; Salo, E.; Yip, R. W.; Chem. Phys. Lett. 1984, 104, 373.

17. Lewis, G. N.; Magel, T. T.; Lipkin, D.; J. Am. Chem. Soc. 1942, 64, 1774 .

18. Lewis, L. M.; Indig, G. L.; Dyes Pigm. 2000, 46, 145.

19. Oliveira, C. S.; Branco, K. P.; Baptista, M. S.; Indig, G. L.; Spectrochim. Acta, Part A 2002, 58, 2971.

20. Maruyama, Y.; Ishikawa, M.; Satozono, H.; J. Am. Chem. Soc. 1996, $118,6257$.

21. Maruyama, Y.; Magnin, O.; Satozono, H.; Ishikawa, M.; J. Phys. Chem. A 1999, 103, 5629.

22. Lovell, S.; Marquardt, B. J.; Kahr, B.; J. Chem. Soc., Perkin Trans. 2 1999, 2241.
23. Angeloni, L.; Smulevich, G.; Marzocchi, M. P.; J. Raman Spectrosc. 1979, 8, 305.

24. Angeloni, L.; Smulevich, G.; Marzocchi, M. P.; J. Mol. Struct. 1980, 61, 331.

25. Lueck, H. B.; Daniel, D. C.; McHale, J. L.; J. Raman Spectrosc. 1993, 24, 363.

26. Loison, C.; Antoine, R.; Broyer, M.; Dugourd, P.; Guthmuller, J.; Simon, D.; Chem. - Eur. J. 2008, 14, 7351.

27. Lee, C. T.; Yang, W. T.; Parr, R. G.; Phys. Rev. B 1988, 37, 785.

28. Frisch, M. J. T.; Trucks, G. W.; Schlegel, H. B.; Scuseria, G. E.; Robb, M. A.; Cheeseman, J. R.; Scalmani, G.; Barone, V.; Mennucci, B.; Petersson, G. A.; Nakatsuji, H.; Caricato, M.; Li, X.; Hratchian, H. P.; Izmaylov, A. F.; Bloino, J.; Zheng, G.; Sonnenberg, J. L.; Hada, M.; Ehara, M.; Toyota, K.; Fukuda, R.; Hasegawa, J.; Ishida, M.; Nakajima, T.; Honda, Y.; Kitao, O.; Nakai, H.; Vreven, T.; Montgomery Jr., J. A.; Peralta, J. E.; Ogliaro, F.; Bearpark, M. J.; Heyd, J.; Brothers, E. N.; Kudin, K. N.; Staroverov, V. N.; Kobayashi, R.; Normand, J.; Raghavachari, K.; Rendell, A. P.; Burant, J. C.; Iyengar, S. S.; Tomasi, J.; Cossi, M.; Rega, N.; Millam, N. J.; Klene, M.; Knox, J. E.; Cross, J. B.; Bakken, V.; Adamo, C.; Jaramillo, J.; Gomperts, R.; Stratmann, R. E.; Yazyev, O.; Austin, A. J.; Cammi, R.; Pomelli, C.; Ochterski, J. W.; Martin, R. L.; Morokuma, K.; Zakrzewski, V. G.; Voth, G. A.; Salvador, P.; Dannenberg, J. J.; Dapprich, S.; Daniels, A. D.; Farkas, Ö.; Foresman, J. B.; Ortiz, J. V.; Cioslowski, J.; Fox, D. J.; Gaussian 09; Gaussian, Inc.: Wallingford, CT, USA, 2009.

29. Marenich, A. V.; Cramer, C. J.; Truhlar, D. G.; J. Phys. Chem. B 2009, $113,4538$.

30. Welton, C. R. A. T.; Solvents and Solvent Effects in Organic Chemistry, $4^{\text {th }}$ ed., Wiley-VCH Verlag \& Co.: KGaA: Weinheim, 2011.

31. Sunder, S.; Bernstein, H. J.; Can. J. Chem. 1981, 59, 964.

FAPESP helped in meeting the publication costs of the article 